\title{
Upregulated ankyrin repeat-rich membrane spanning protein contributes to tumour progression in cutaneous melanoma
}

\author{
YH Liao*,', SM Hsu', ${ }^{2,3}$ HL Yang', MS Tsai ${ }^{3}$ and PH Huang,2,3 \\ 'Department of Dermatology, National Taiwan University Hospital, College of Medicine, National Taiwan University, No. 7, Chung-Shan South Road, Taipei 100, \\ Taiwan; ' ${ }^{2}$ epartment of Pathology, National Taiwan University Hospital, College of Medicine, National Taiwan University, No. 7, Chung-Shan South Road, \\ Taipei 100, Taiwan; ${ }^{3}$ Graduate Institute of Pathology, College of Medicine, National Taiwan University, No. I, Sec. I, Ren-Ai Road, Taipei I00, Taiwan
}

BACKGROUND: We have previously demonstrated that overexpression of ankyrin repeat-rich membrane spanning (ARMS) protein facilitates melanoma formation via conferring apoptotic resistance. This study aims to investigate whether ARMS contributes to melanoma progression. METHOD: Using immunohistochemistry, we graded the expression level of ARMS in 54 cases of primary melanoma and 46 cases of metastatic melanoma. The immunointensity of ARMS was statistically correlated with individual clinicopathological characteristics. By RNA interference, stable melanoma cell clones with ARMS-knockdown were constructed, and were used for in vitro scratch wound, transwell invasion assays, and in vivo lung metastasis experiment.

RESULTS: Stronger immunointensity of ARMS was observed mostly in melanomas with Breslow tumour thickness $>1.0$ mm (Fisher's exact test, $P=0.002$ ) or with nodal metastasis (Fisher's exact test, $P=0.026$ ), and was correlated with a worse overall survival in melanoma patients (log-rank test, $P=0.04$ ). Depletion of ARMS inhibited migration, invasion, and metastatic potential of melanoma cells in vitro and in vivo. Moreover, ARMS mediated melanoma cell migration and invasion through activation of the extracellular signal-regulated kinase (ERK) kinase (MEK)/ERK signalling pathway.

CONCLUSION: Ankyrin repeat-rich membrane spanning expression, conjunctly with tumour thickness or ulceration, may serve as a prognostic factor in patients with cutaneous melanoma.

British Journal of Cancer (201I) 1 04,982-988. doi:10.1038/bjc.201 I.18 www.bjcancer.com

Published online 22 February 2011

(c) 201 I Cancer Research UK

Keywords: ARMS; cutaneous melanoma; tumour thickness; melanoma progression; MEK/ERK

Cutaneous malignant melanoma is a highly aggressive cancer with poor prognosis at advanced stage. It originates from malignant transformation of melanocytes or nevocytes. During tumour progression from radial growth phase to invasive vertical growth phase, melanoma cells acquire metastatic capability and are usually resistant to current chemotherapy (Miller and Mihm, 2006). Several histological factors have been shown to correlate with the survival of melanoma patients, among which Breslow tumour thickness is recognised as the most important prognostic parameter (Sober et al, 1979). Although patients with thin melanomas have an excellent prognosis following curative surgery, those patients with increased thickness of the lesions $(>1.0 \mathrm{~mm}$ Breslow tumour thickness) are at risk for distant metastasis and poor survival (Balch et al, 2001, 2009).

Reflecting its ontogenic origin as neural crest cells, melanoma frequently re-expresses molecules functioning in neuronal development. For example, muscarinic acetylcholine receptors, neuron-specific enolase, and microtubule-associated protein 2 are overexpressed in malignant melanomas (Dhillon and Rode, 1982; Lammerding-Koppel et al, 1997; Soltani et al, 2005). We have demonstrated that ARMS (ankyrin repeat-rich membrane

*Correspondence: Dr YH Liao or Dr PH Huang;

E-mail: yihualiao@ntu.edu.tw or phhuang@ntu.edu.tw

Received 8 December 20 10; revised 4 January 201 I; accepted 10 January 20I I; published online 22 February 20I I spanning), a neuron-enriched transmembrane molecule interacting with Trk, p75 ${ }^{\mathrm{NTR}}, \mathrm{Eph}$, and protein kinase D (Iglesias et al, 2000; Kong et al, 2001; Chang et al, 2004), is overexpressed in cutaneous melanoma, while normal melanocytes and benign nevi reveal negative or weak expression of ARMS (Liao et al, 2007). Expression of ARMS in melanoma cells can confer resistance to UVB-induced apoptosis through activating the MEK/extracellular signal-regulated kinase (ERK) signalling pathway and thus facilitates neoplastic transformation (Liao et al, 2007). In this study, we investigated the role of ARMS in the malignant progression of cutaneous melanoma. We analysed and statistically correlated the immunointensity of ARMS in archived tissues with the clinicopathological characteristics of patients with cutaneous melanoma. Besides, statistical analysis was performed to correlate the expression level of ARMS with the patient overall survival. We further used RNA interference (RNAi) to knock down the expression of ARMS in mouse and human melanoma cell lines, and explored whether ARMS regulated the migratory/invasive ability of melanoma cells via in vitro and in vivo assays.

\section{MATERIALS AND METHODS}

\section{Antibodies}

Polyclonal anti-ARMS antibody was generated as described (Liao et al, 2007). 


\section{Case selection}

A total of 100 melanoma lesions were selected by searching the pathological records from 1997 to 2006 registered in National Taiwan University Hospital (NTUH). Tissue blocks were derived from the archives of Department of Pathology, NTUH, following the guidelines set forth by Tissue Committee at NTUH. Selected demographic information, including patient age, gender, tumour location, tumour subtype, and outcome of survival was retrieved from the hospital cancer registry.

\section{Tissue microarray and immunohistochemistry}

Tissue microarrays were constructed from archival formalin-fixed, paraffin-embedded surgical specimens registered at Department of Pathology, NTUH. Sampling of tumour regions was based on visual alignment with the corresponding haematoxylin-eosinstained sections. Tissue cores were placed into a recipient paraffin block with a tissue microarrayer (Manual Tissue Arrayer 1; Beecher Instruments, Inc., Sun Prairie, WI, USA), and 5- $\mu \mathrm{m}$ sections of the resulting microarray block were used for immunohistochemistry. Immunohistochemistry for ARMS staining was performed as previously described (Liao et al, 2007). The sections were examined, and the staining intensity of ARMS was scored by two pathologists.

\section{Cell culture, RNAi, and selection of stable-transfected clones}

Mouse B16-F0, -F1, -F10 and human A2058, A375 melanoma cell lines were from the American Type Tissue Culture Collection (ATCC; Manassas, VA, USA) and maintained in DMEM supplemented with $10 \% \mathrm{FBS}, 4 \mathrm{~mm}$ L-glutamine, $100 \mu \mathrm{g} \mathrm{ml}^{-1}$ streptomycin, and $100 \mathrm{U} \mathrm{ml}^{-1}$ penicillin. The siRNA expression vector pSUPER.neo + GFP (OligoEngine, Seattle, WA, USA) was used for expression of ARMS siRNA or control scrambled siRNA in B16F10 cells. The specific sequence for paired RNA oligonucleotides contained nucleotides 4271-4289 for ARMS-RNAi-1 and 48404858 for ARMS-siRNA-2 in mouse ARMS (accession number: AK122478). Transfection and selection of ARMS knockdown stable cell clones were performed as previously described (Liao et al, 2007). The RNAi rescue experiment was performed by transfecting the ARMS-RNAi-2/B16-F10 stable clone with the ARMSpcDNA3.1C-Myc $4849 \mathrm{~T} \rightarrow \mathrm{C}$ expression mutant as previously described (Liao et al, 2007). Lentivirus carrying ARMS-specific and scrambled small interfering RNA were purchased from the National RNAi Core Facility of Academia Sinica (Taipei, Taiwan). The sequences used for ARMS targeting was $5^{\prime}$-GCACTGATTG TGGCAGTGAAA-3'. To generate recombinant lentivirus, 293FT cells were co-transfected with the package, envelop, and siRNAexpressing constructs. The virus-containing supernatant was harvested and then used to infect A2058 and A375 human melanoma cells. The infected cells were selected with puromycin $2 \mu \mathrm{g} \mathrm{ml}^{-1}$ and the stable clones with the highest knockdown efficiency were used for subsequent experiments.

\section{Invasion assays}

The invasiveness of melanoma cells were assayed using Boyden chambers containing polycarbonate filters (Transwell $24,8-\mu \mathrm{m}$ pore; Costar, Cambridge, MA, USA). Filters were coated with $2 \mathrm{mg} \mathrm{ml}^{-1}$ Matrigel (Becton Dickinson, Heidelberg, Germany) at $37^{\circ} \mathrm{C}$ overnight. Cells were harvested, resuspended in medium, and placed in the upper wells at a density of $5 \times 10^{4}$ in $100 \mu$ l DMEM. In some cases, $10 \mu \mathrm{M}$ PD98059 (Calbiochem, La Jolla, CA, USA) or dimethyl sulfoxide (DMSO; $0.02 \%$ ) was added to the upper and lower chambers. After incubation for $48 \mathrm{~h}$ at $37^{\circ} \mathrm{C}$, membrane toward upper chamber is scraped by cotton swabs. The cells adhering to the lower surface were pre-fixed with $4 \%$ paraformaldehyde, stained with crystal violet, and counted by microscope observation. For each membrane, the mean number of cells of five randomly selected fields $(100 \times)$ in the centre of the filter was obtained. The experiments were performed in triplicate.

\section{Scratch wound assay}

Melanoma cells were seeded in high density $\left(5 \times 10^{5}\right)$ into $35 \mathrm{~mm}$ dishes. Scratch wounds were made by creating three linear denuded regions using a pipette tip per dish. The cells were washed twice with medium before their incubation. In some cases, $10 \mu \mathrm{M}$ PD98059 or DMSO $0.02 \%$ was added in the medium. Six fields per dish were photographed at 0 and $17 \mathrm{~h}$. Migration into the wounds was documented and measured by MetaMorph7.6 Software (Molecular Devises Inc., Sunnyvale, CA, USA). Each analysis was performed in triplicate.

\section{In vivo metastasis assay}

Control and ARMS - RNAi stable B16-F10 cell lines were individually injected into C57BL/6J mice via the lateral tail vein $\left(5 \times 10^{5}\right.$ cells per mouse, $n=5$ in each group). Mice were killed 10 days after intravenous injection. The lungs were fixed, photographed, and the number of visible black nodules on the lung surface was counted with a dissecting microscope. The lung tissue was then embedded in paraffin and sectioned at three different levels; the sections were stained with haematoxylin and eosin for light microscopy.

\section{Statistical analysis}

The correlation between the intensity of ARMS expression and the clinicopathological parameters was evaluated by two-sided Fisher's exact test. The impact of immunointensity of ARMS, tumour thickness, ulceration, tumour location, age, and sex on patient overall survival was assessed by the Kaplan-Meier method, and differences between groups were analysed by the log-rank test. Multivariate Cox proportional hazard regression analysis was used to assess independent variables. $P<0.05$ were considered statistically significant. Statistics were performed with the SPSS 12.0 software (SPSS Inc., Chicago, IL, USA) and calculations on patient survival were performed using the GraphPad Prism software (GraphPad Software Inc., San Diego, CA, USA).

\section{RESULTS}

Overexpression of ARMS in primary and metastatic melanoma with enhanced immunoreactivity at tumour invasive fronts

We examined ARMS expression in tumours of different tissue type by immunohistochemistry on tumour tissue arrays. In this screening, ARMS was specifically expressed in tumours of neuroepithelium origin, which included pheochromocytoma, paraganglioma, central neurocytoma, and malignant melanoma (Supplementary Figure S1A). Other epithelial-derived tumours had barely or none detectable ARMS, which included 13 breast cancers (6 ductal, 2 lobular, 3 medullary, 1 papillary, and 1 metaplastic carcinoma), 12 lung cancers (5 squamous cell carcinoma and 7 adenocarcinoma), 6 gastric adenocarcinoma, 7 colon adenocarcinoma, 5 pancreatic adenocarcinoma, and 5 hepatocellular carcinoma (Supplementary Figures S1B-F). For skin cancers, expression of ARMS was specific to malignant melanoma in that non-melanocytic skin cancers such as basal cell carcinoma $(n=5)$, squamous cell carcinoma $(n=5)$, and extramammary Paget's disease $(n=3)$ did not express ARMS (Supplementary 
Figures S1G-I). The results indicate that ARMS is specifically expressed in tumours of neuroectodermal origin.

On the basis of the results from the immunohistochemistry screening of ARMS in tumour tissue arrays, we collected 100 cases of primary cutaneous melanoma and metastatic melanoma for further study. In accordance with our previous report (Liao et al, 2007), positive immunoexpression of ARMS was detected in $94.4 \%$ of cases with primary malignant melanoma as well as in $87 \%$ of cases with metastatic melanomas (Table 1). For primary melanoma, those ARMS-negative cases were all in situ lesions (Figure 1A).

Table I ARMS expression in primary cutaneous melanoma and metastatic melanoma $(n=100)$

\begin{tabular}{llllllll} 
& \multicolumn{5}{c}{ ARMS immunostain intensity } & \\
\cline { 2 - 6 } Lesion & $\boldsymbol{n}$ & Negative & Weak & Moderate & Strong & $\boldsymbol{P}_{\text {-value }}$ \\
\hline Primary & 54 & $3(5.6 \%)$ & $20(37.0 \%)$ & $21(38.9 \%)$ & $10(18.5 \%)$ & \\
melanoma & & & & & & \\
Metastatic & 46 & $6(13.0 \%)$ & $17(37.0 \%)$ & $14(30.4 \%)$ & $9(19.6 \%)$ & 0.58 \\
melanoma & & & & & & \\
\hline
\end{tabular}

Abbreviation: ARMS $=$ ankyrin repeat-rich membrane spanning. ${ }^{a} \chi^{2}$-test.
When the immunointensity of ARMS was further graded into weak, moderate, and strong intensity, stronger immunoreactivity of ARMS was found at the invasive front of tumour nests facing toward the dermis (Figures $1 \mathrm{~B}$ and $\mathrm{C}$, arrowheads) and in tumour cells invading the nerves (Figure 1D). These observations suggest that ARMS might be involved in the processes mediating local tumour invasiveness. However, when the immunointensity of ARMS was compared between metastatic melanoma and primary melanoma, no statistical significance was observed (Fisher's exact test, $P=0.58$; Table 1 ). Immunohistochemistry of ARMS demonstrated two staining patterns: cytoplasmic granular and membranous distribution (Figures $1 \mathrm{E}$ and $\mathrm{F}$ ). Both patterns were seen in primary cutaneous and metastatic melanoma with a similar percentage.

\section{The immunointensity of ARMS correlates with tumour thickness in cutaneous melanoma}

The clinical outcome of patients with cutaneous melanoma is influenced by several factors including age, gender, ulceration, tumour subtype, and in particular, tumour thickness (Balch et al, 2001). To examine whether ARMS expression in cutaneous melanoma was associated with any clinicopathological parameter,
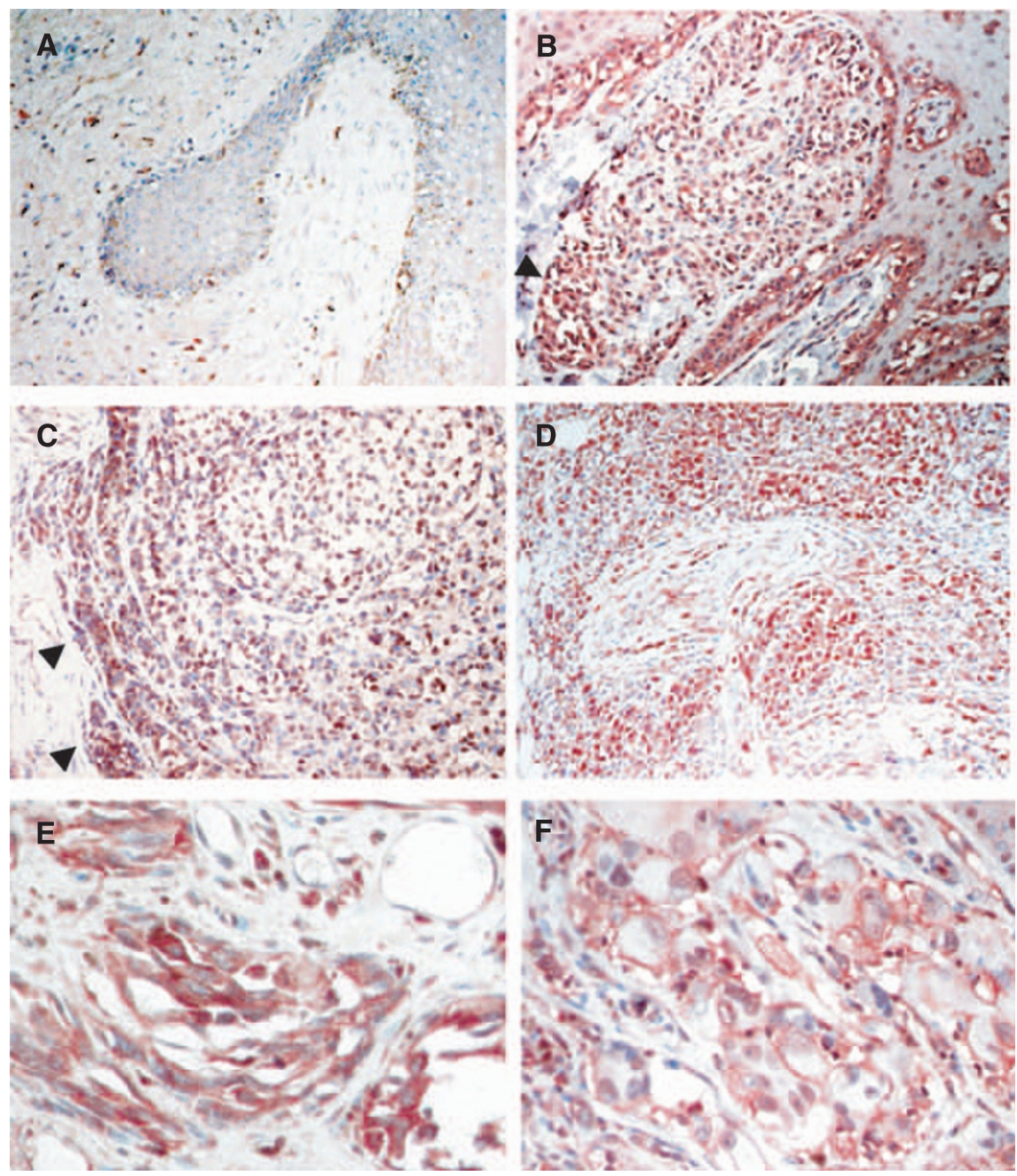

Figure I Immunohistochemical analysis of ARMS in cutaneous melanoma. Representative immunohistochemistry of ARMS in melanoma in situ and melanoma at vertical growth phase. Ankyrin repeat-rich membrane spanning staining was negative in melanoma in situ ( $\times 200$, A). Stronger immunointensity of ARMS was shown at the invasive front $(\times 200$, B and $\mathbf{C}$, arrow heads $)$ and in tumour cells invading the nerve in the dermis at vertical growth phase $(\times 100, \mathbf{D})$. Either cytoplasmic granular $(\times 400, \mathbf{E})$ or membranous $(\times 400, \mathbf{F})$ distribution pattern of ARMS was observed in cutaneous melanoma. 
Table 2 Statistical correlations between the immunointensity of ARMS and each clinicopathological characteristic in 54 patients with primary cutaneous melanoma

\begin{tabular}{|c|c|c|c|c|c|c|}
\hline & \multicolumn{4}{|c|}{ Intensity of ARMS staining } & \multirow[b]{2}{*}{ Total } & \multirow[b]{2}{*}{$P$-value } \\
\hline & Negative & Weak & Moderate & Strong & & \\
\hline \multicolumn{7}{|l|}{ Age } \\
\hline$\leqslant 64$ & $2(7.4 \%)$ & II (40.8\%) & $10(37.0 \%)$ & $4(14.8 \%)$ & 27 & 0.86 \\
\hline$>64$ & I (3.7\%) & $9(33.3 \%)$ & $12(44.4 \%)$ & $5(18.5 \%)$ & 27 & \\
\hline \multicolumn{7}{|l|}{ Sex } \\
\hline Male & $2(7.7 \%)$ & $10(38.5 \%)$ & $8(30.8 \%)$ & $6(23.1 \%)$ & 26 & 0.098 \\
\hline Female & | (3.6\%) & $10(35.7 \%)$ & $14(50 \%)$ & $3(10.7 \%)$ & 28 & \\
\hline \multicolumn{7}{|c|}{ Tumour thickness (mm) } \\
\hline$\leqslant 1.0$ & $3(20.0 \%)$ & $2(13.3 \%)$ & $10(66.7 \%)$ & $0(0 \%)$ & 15 & $0.002^{b}$ \\
\hline$>1.0$ & $0(0.0 \%)$ & $18(46.1 \%)$ & $12(30.8 \%)$ & $9(23.1 \%)$ & 39 & \\
\hline \multicolumn{7}{|l|}{ Ulceration } \\
\hline Absent & $3(7.7 \%)$ & $16(41.0 \%)$ & $17(43.6 \%)$ & $3(7.7 \%)$ & 39 & 0.053 \\
\hline Present & $0(0.0 \%)$ & $4(26.7 \%)$ & $5(33.3 \%)$ & $6(40.0 \%)$ & 15 & \\
\hline \multicolumn{7}{|c|}{ Lymph node invasion or distant metastasis } \\
\hline Negative & $3(12.5 \%)$ & $6(25.0 \%)$ & $13(54.2 \%)$ & $2(8.3 \%)$ & 24 & $0.026^{\mathrm{b}}$ \\
\hline Positive & $0(0.0 \%)$ & $14(46.7 \%)$ & $9(30.0 \%)$ & $7(23.3 \%)$ & 29 & \\
\hline \multicolumn{7}{|c|}{ Tumour subtype } \\
\hline ALM & $3(7.1 \%)$ & $15(35.7 \%)$ & $18(42.9 \%)$ & $6(14.3 \%)$ & 42 & 0.44 \\
\hline NM & $0(0.0 \%)$ & $3(60.0 \%)$ & $0(0 \%)$ & $2(40.0 \%)$ & 5 & \\
\hline SSM & $0(0.0 \%)$ & $2(28.6 \%)$ & $4(57.1 \%)$ & I (14.3\%) & 7 & \\
\hline
\end{tabular}

Abbreviations: $A L M=$ acral lentiginous melanoma: $A R M S=$ ankyrin repeat-rich membrane spanning; $N M=$ nodular melanoma; $\quad S S M=$ superficial spreading melanoma. ${ }^{a}$ Fisher's exact test; ${ }^{b} p<0.05$.

we correlated each demographic characteristic with the immunointensity of ARMS among the 54 patients with primary cutaneous melanoma (Table 2 ). In this study group, 42 cases $(77.8 \%)$ were diagnosed as acral lentiginous melanoma, 5 cases $(9.3 \%)$ as nodular melanoma, and 7 cases as superficial spreading melanoma $(13 \%)$. There were 26 men and 28 women included in this study group, and their age ranged from 28 to 88 years old (the median, 64 years). We used Breslow tumour thickness to evaluate local invasion of melanoma. By this way, 15 cases $(27.8 \%)$ were classified as the subgroup with lesion $\leqslant 1.0 \mathrm{~mm}$, and the remaining 39 cases $(72.2 \%)$ were within the subgroup with lesion $>1.0 \mathrm{~mm}$.

Analysis of ARMS expression and the clinical parameters including age, gender, ulceration, and tumour subtypes did not reveal any significant association (Table 2). However, when the immunointensity of ARMS was compared in terms of Breslow thickness, melanomas with tumour thickness $>1.0 \mathrm{~mm}$ tended to have stronger ARMS immunoreactivity than lesions of melanoma in situ. Positive ARMS staining was detected in all cases of melanoma with thickness $>1.0 \mathrm{~mm}$, of which $23.1 \%$ were strongly stained. Whereas for thin melanomas (thickness $<1.0 \mathrm{~mm}$ ), $20 \%$ was nonreactive to ARMS, $80 \%$ showed weak-to-moderate ARMS immunointensity, and none of them showed strong immunoreactivity toward ARMS (Fisher's exact test, $P=0.002$; Table 2). When the immunointensity of ARMS was compared between tumours with and without nodal/distant metastasis, $23.3 \%$ of tumours with nodal metastasis showed strong ARMS expression, compared with $8.3 \%$ of those without nodal metastasis (Fisher's exact test, $P=0.026$; Table 2). Combined all together, it suggests that ARMS expression is strongly upregulated in the cutaneous melanomas with tumour thickness $>1.0 \mathrm{~mm}$, and overexpression of ARMS may be related to local dermal invasion and distant nodal metastasis.

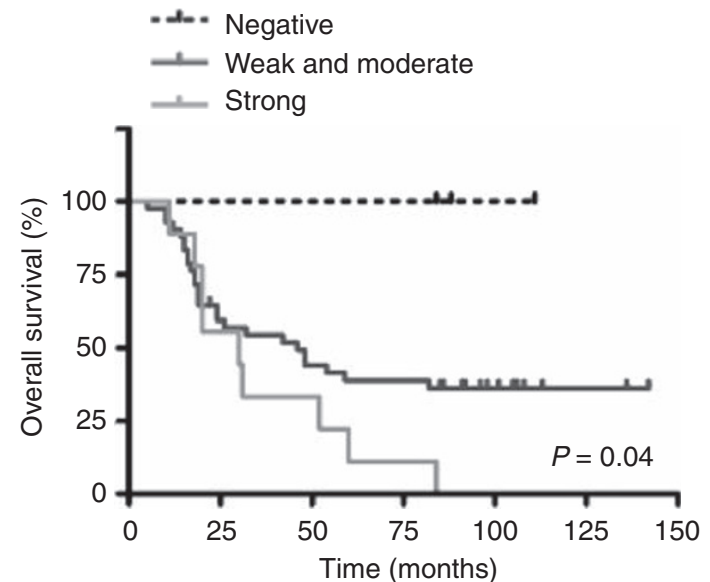

Figure 2 Kaplan-Meier survival analysis between ARMS expression and the overall survival in melanoma patients. Patients with negative-, weak-, or moderate-ARMS expression have a significantly better overall survival than those with strong-ARMS expression ( $P<0.05$, log-rank test).

\section{Prognostic relevance of ARMS expression}

To evaluate whether the expression of ARMS in primary cutaneous melanomas was related to patient outcome, a Kaplan-Meier survival curve was constructed. The influence of tumour thickness and ulceration, two well-recognised prognostic factors on overall survival for cutaneous melanoma (Balch et al, 2009), was also assessed in this study group as a method standard and a comparison. Our analysis showed that both Breslow thickness (log-rank test, $P=0.001$ ) and tumour ulceration (log-rank test, $P=0.007)$ were significant prognostic factors in our patients with primary cutaneous melanoma. Importantly, a significant correlation between the immunointensity of ARMS and the patient overall survival was shown (log-rank test, $P=0.04$; Figure 2). Shorter median survival time in melanoma patients with stronger ARMS expression (30 months) was revealed in comparison with the patients with weak-to-moderate ARMS immunointensity (42 months). Therefore, the expression level of ARMS may serve as a molecular predictor for the prognosis of patients with primary cutaneous melanoma.

To investigate whether the immunointensity of ARMS was an independent prognostic marker for melanoma, we applied a multivariate Cox's proportional hazards model for the assessment of the overall survival. The variables taken into consideration included age, sex, tumour thickness, ulceration, tumour location, and intensity of ARMS staining. Such analysis demonstrated that only tumour thickness retained its significant predictive value for the overall survival $(P=0.03$, Supplementary Table 1). While comparing patients with strong immunointensity to those with negative, weak, or moderate ARMS staining, the hazard ratio was $1.28(95 \%$ confidence interval $0.446-3.69, P=0.64)$ without reaching statistical significance (Supplementary Table 1).

\section{ARMS-knockdown diminishes migratory and invasive abilities of melanoma cells in vitro and in vivo through downregulation of MEK/ERK activity}

To investigate the role of ARMS in tumour invasion and migration, we first examined the expression of ARMS in a panel of B16 melanoma cell lines with differential migratory ability. Western blot analysis revealed a trend of progressively increased ARMS expression associated with enhanced cell migratory ability. As shown in Figure 3A, ARMS protein was expressed at low levels in the benign mouse melanocyte cell line Melan-A (set as 1 for comparison), and was moderately increased in the less metastatic 
A

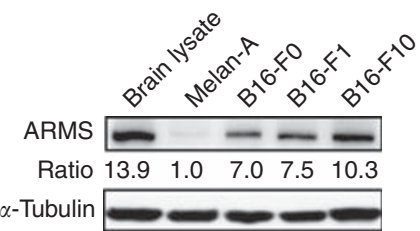

B

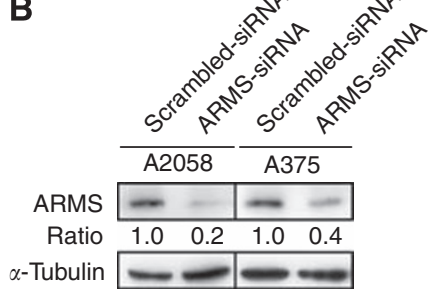

C

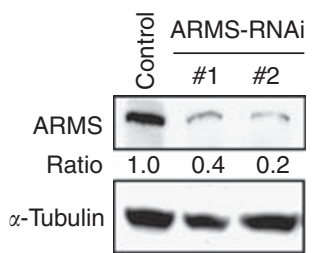

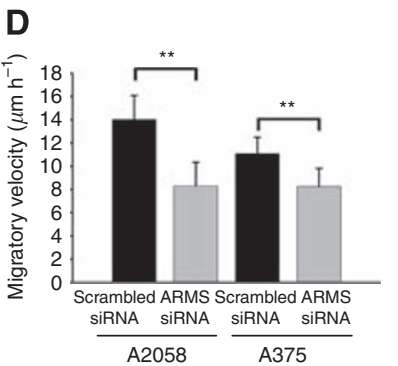

E
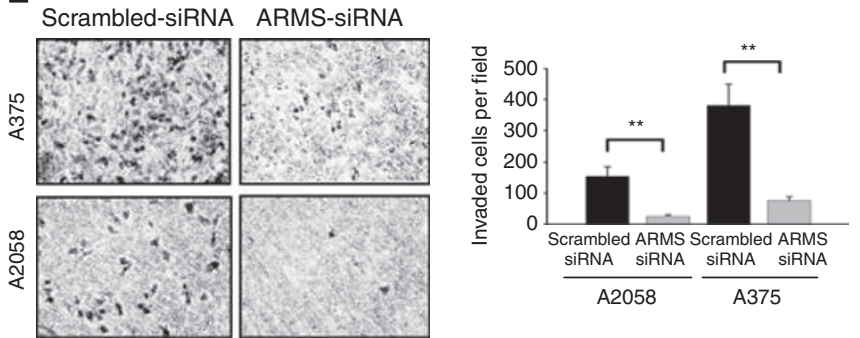

$\mathbf{F}$

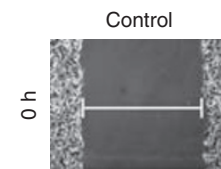

ARMS-RNAi
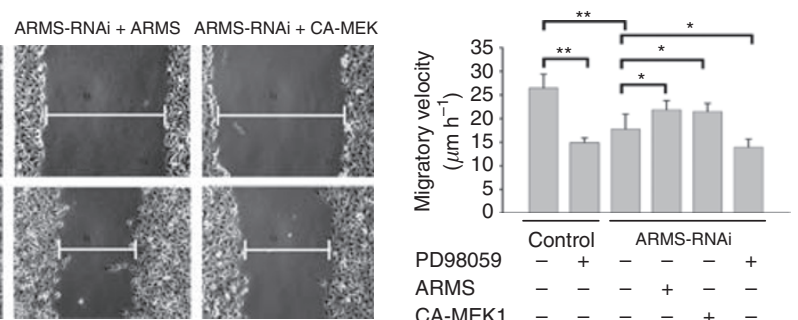

G
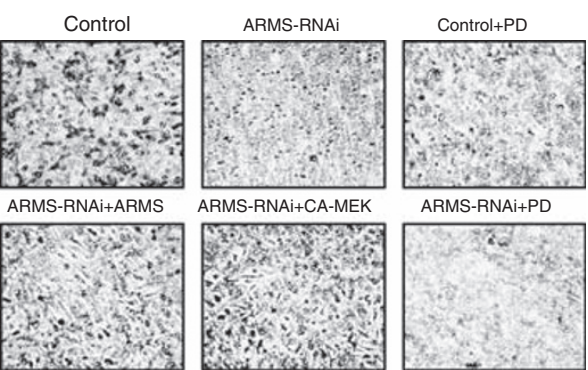

I Contro

CA-MEK1 - $-\ldots++$
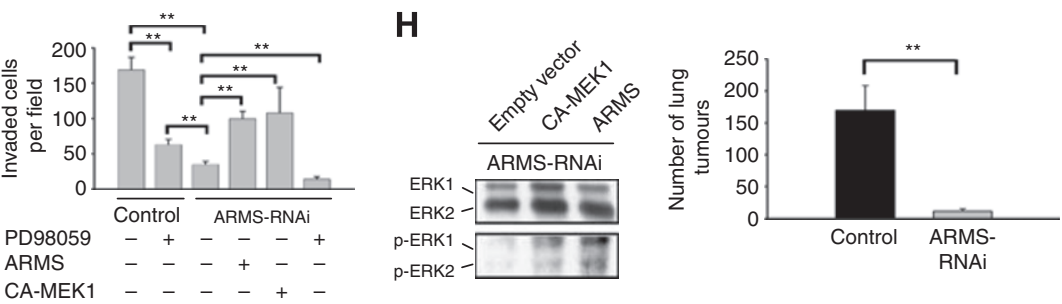

Figure 3 Depletion of ARMS deteriorates migratory and invasive abilities in melanoma cells via MEK/ERK signalling. (A) Western blot and quantification of ARMS protein levels in mouse melanoma BI6-FO,-FI, and - $\mathrm{FIO}$ cell lines as normalised to $\alpha$-tubulin using densiometry showed increased expression of ARMS in BI6-FIO cells, the most aggressive melanoma cell line among them. Melan-A cells were given a value of I for comparison. Mouse brain lysate was used as a positive control. (B) Western blotting showed effective ARMS knockdown by siRNA in human A2058 and A375 melanoma cells. (C) Effective RNAi of endogenous ARMS in two independent mouse BI6-FIO ARMS-RNAi stable cell clones as compared with their controls (scrambled). Levels of $\alpha$-tubulin were shown as a loading control. (D and E) ARMS knockdown in human A2058 and A375 melanoma cells resulted in a reduction of migration velocity (bar graph, means \pm s.d.) in a wound-scratch assay (D) and decreased the invasive ability of the cells in a Boyden chamber invasion assay (E). Representative photomicrographs with the cresyl violet stain and bar graph (means \pm s.d.) demonstrated the status of cellular invasion between the control and ARMS-RNAi cells in a Boyden chamber invasion assay (magnification $\times 200$ ). The experiments were performed in triplicate. $* * * 20.0$ I, Student's $t$-test. $(\mathbf{F}$ and $\mathbf{G})$ ARMS silencing leaded to a significant reduction of migratory and invasive ability of BI6-FIO cells via MEK/ERK signalling. Forced expression of RNAi-resistant ARMS or constitutively active MEK-I (CA-MEKI) into BI6-FIO melanoma cells with ARMS-knockdown resulted in a significant increase in cellular migratory ability (F, representative images and bar graph) and invasive potential in a Boyden chamber invasion assay (G, representative images and bar graph). Inhibition of MEK/ERK by PD98059 treatment in control and ARMS-RNAi cells compromised cell migration (F, bar graph) and invasion (G, images and bar graph). *P $<0.05$; ** $P<0.0$ I; Student's $t$-test. $(\mathbf{H})$ Overexpression of RNAi-resistant ARMS or CA-MEKI in ARMS-RNAi cells resulted in ERK I/2 phosphorylation in melanoma cells compared with ARMS-RNAi cells. (I) The number of lung metastatic melanoma nodules was significantly decreased in mice receiving intravenous injection of ARMS - RNAi melanoma cells compared with that of control mice. Tumour nodules were shown in representative grossly dissected lungs (upper panels) and mouse lung tissue sections stained with haematoxylin and eosin (lower panels, $\times 200$ ) in each group. *** $<0.0$ l, Student's $t$-test. 
B16-F0 cells and B16-F1 cells (7-fold and 7.5-fold increase, respectively). By contrast, in highly metastatic B16-F10 melanoma cells, high levels of ARMS expression were observed (10.3-fold increase). When immunofluorescence was performed in human melanoma cell lines, colocalisation of ARMS with actin and relative enhancement of ARMS in invading filopodia or lamellipodia were observed (Supplementary Figure S2). Taken together, these results suggest that ARMS may be involved in melanoma progression by regulating cell migration and invasion.

To explore the cause-effect relationship between ARMS expression and melanoma aggressiveness, we used RNAi to knockdown ARMS expression in three metastatic melanoma cell lines with abundant ARMS expression (human A2058, human A375, and mouse B16-F10 cells). Expression of ARMS in human A2058 and A375 cells was silenced to the level around 20 and $40 \%$, respectively, of that in control scrambled cells (Figure 3B). For mouse B16-F10 melanoma cells, two independent clones targeted by different nucleotide sequences (designated as ARMS-RNAi-1 and ARMS-RNAi-2) had ARMS expression to the level around 40 and $20 \%$ of that in control, respectively (Figure 3C). The proliferation rate of these constructed stable clones was not affected by ARMS silencing, as evidenced by Trypan blue exclusive assay (data not shown).

The migratory and invasive capacities of melanoma cells with or without ARMS - RNAi were assessed by in vitro wound scratch and chamber invasion assays. As shown in Figure 3D, ARMS silencing decreased migratory velocity in both A2058 and A375 cells. The mean migratory velocity in A2058 cells was $8.3 \pm 2.0 \mu \mathrm{m} \mathrm{h}^{-1}$ for ARMS - RNAi vs $14.0 \pm 2.1 \mu \mathrm{m} \mathrm{h}^{-1}$ for control cells, and in A375 cells was $8.2 \pm 1.6 \mu \mathrm{m} \mathrm{h}^{-1}$ for ARMS-RNAi $v s 11.1 \pm 1.4 \mu \mathrm{m} \mathrm{h}^{-1}$ for control cells (Student's $t$-test, $P<0.01$ ). Knockdown of ARMS also decreased invasion capacity of A2058 (mean \pm s.d.; $154.0 \pm 31.4$ cells per $100 \times$ field in control cells $v s 25.3 \pm 4.7$ cells in ARMSRNAi cells) and A375 cells ( $380.7 \pm 68.5$ cells in control cells $v s$ $76.2 \pm 12.5$ cells in ARMS - RNAi cells) as evidenced by Boyden chamber invasion assay (Figure $3 \mathrm{E}$; Student's $t$-test, $P<0.01$ ). Similarly, ARMS silencing in mouse B16-F10 melanoma cells significantly demised the migration potential of tumour cells in wound scratch assay $\left(17.8 \pm 3.1 \mu \mathrm{mh}^{-1}\right.$ for ARMS-RNAi $v s$ $26.5 \pm 2.9 \mu \mathrm{m} \mathrm{h}^{-1}$ for control) and led to a significant decrease in their invasive capability $(169 \pm 17.6$ cells per $100 \times$ field in control cells $v s 35 \pm 4.4$ cells in ARMS - RNAi cells; Figure 3F; Student's $t$-test, $P<0.01)$. Reintroduction of RNAi-resistant full-length ARMS into ARMS-knockdown cells significantly recovered the compromised migratory and invasive capability of melanoma cells resulting from ARMS depletion as evident by wound scratch $\left(21.6 \pm 2.0 \mu \mathrm{m} \mathrm{h}^{-1}, 43.5 \%\right.$ recovery compared with ARMS - RNAi cells; Figure $3 \mathrm{~F})$ and by the chamber invasion assay $(99.7 \pm 10.5$ cells per $100 \times$ field, $49.3 \%$ recovery compared with ARMS - RNAi cells; Figure 3G). These results suggest that knockdown of ARMS significantly decreases the migratory and invasive potential of human A2058, A375, and murine B16-F10 melanoma cells.

Given that MEK/ERK molecules are downstream effectors of ARMS, we thus examined the role of MEK/ERK signalling in ARMS-mediated migration and invasion. First, we introduced constitutively active MEK1 (CA-MEK1) into ARMS-knockdown cells and observed that the compromised migratory and invasive capability of melanoma cells resulting from ARMS depletion was recovered (Figures $3 \mathrm{~F}$ and $\mathrm{G}$ ). Second, treatment of B16-F10 control cells with a MEK-specific inhibitor PD98059 resulted in a significant decrease in migratory and invasive ability similar to that in ARMS-RNAi cells (Figures $3 \mathrm{~F}$ and G). Third, treatment of ARMS - RNAi cells with PD98059 further inhibited cell migration and invasion in comparison with vehicle-treated ARMS-RNAi cells (Figures $3 \mathrm{~F}$ and $\mathrm{G}$ ). Western blotting confirmed that both phospho-ERK1/2 were markedly upregulated by expression of CA-MEK1 and RNAi-resistant ARMS in the ARMS - RNAi cells that had decreased phosphorylated ERKs, while the expression levels of total ERK proteins were not altered (Figure $3 \mathrm{H}$ ). Collectively, these results suggest that MEK/ERK signalling, which is downstream of ARMS regulates the migratory and invasive abilities in melanoma cells.

We then examined whether ARMS depletion interfered with the metastatic behaviour via murine lung metastasis assay. In all, $5 \times 10^{5}$ control or ARMS - RNAi B16-F10 cells were injected into $\mathrm{C} 57 \mathrm{BL} / 6 \mathrm{~J}$ mice via the tail veins, and the animals were assessed for lung metastases 10 days after injection ( $n=5$ in each group). As revealed in Figure 3I, an average of $170 \pm 38.8$ lung tumours developed in mice injected with control scrambled cells. By contrast, only $12 \pm 3.5$ lung tumours were seen in mice injected with ARMS-RNAi melanoma cells (Student's $t$-test, $P<0.01$ ). Combined all together, the in vitro and in vivo data suggest that ARMS silencing deters melanoma cells from invasion and distant metastasis through downregulation of MEK/ERK signalling activity.

\section{DISCUSSION}

In this study, we demonstrate that ARMS is overexpressed in primary melanoma and in metastatic melanoma as well. The immunointensity of ARMS tends to be stronger in melanoma with tumour depth $>1.0 \mathrm{~mm}$, and is intensively localised at the invasive front of melanoma nests in the dermis. Besides, stronger ARMS expression is more frequently found in cases of cutaneous melanoma with lymph node or distant metastasis. Kaplan-Meier analysis further demonstrates that increased ARMS expression correlates negatively with the overall survival of melanoma patients. Therefore, ARMS may serve as a prognostic molecular marker in association with tumour thickness in cutaneous melanoma.

We further demonstrate that ARMS participates in the regulation of migratory/invasive ability of melanoma cells by in vitro and in vivo assays. We provide evidence that ARMS-mediated migratory and invasive abilities depend on the activated MEK/ERK signalling pathway, because constitutively active MEK reverts the compromised migratory and invasive activities caused by ARMS depletion and MEK1 inhibitor PD98059 leads to decreased migratory and invasive potential in melanoma cells. Therefore, activation of the ARMS/MEK/ERK signalling pathway is important for the acquisition of an invasion and metastatic phenotype in melanoma cells. As ARMS forms protein complex with Trk receptor tyrosine kinases or p75 neurotrophin receptor, and can transduce neurotrophin-mediated signalling that leads to ERK activation (Kong et al, 2001; Chang et al, 2004; Arevalo et al, 2004, 2006), it is interesting to identify that expression of neurotrophin, neurotrophin receptors, and phospho-ERK1/2 increases in a stage-specific trend during melanoma progression, just as ARMS does as shown in this study (Brocker et al, 1991; Easty et al, 1995; Walch et al, 1999; Innominato et al, 2001; Shonukan et al, 2003; Smalley, 2003; Zhuang et al, 2005). Similar to the distribution of ARMS at invading fronts of melanoma nests in this study, expression of phospho-ERK and p75NTR is more intense towards the deep tumour margins of thick melanoma (Shonukan et al, 2003; Zhuang et al, 2005). Also in accordance with the positive correlation between ARMS expression and the overall survival in patients with cutaneous melanoma, statistical analysis also shows a worse overall survival for phosphoERK expression in patients with melanoma over $1 \mathrm{~mm}$ in thickness (Zhuang et al, 2005).

It is presently unclear how ARMS regulates migration and invasion in melanoma cells. The colocalisation of ARMS with F-actin on the cell cortex and cell projections in melanoma cells suggests that actin reorganisation could be modulated by ARMS to affect cell motility. Noticeably, it has been reported that p75NTR, an interacting receptor with ARMS, can mediate neurotrophin-dependent melanoma cell migration through specifically interacting with actin-bundling protein fascin (Shonukan et al, 2003). A recent study also reveals that ARMS is concentrated on filopodia and lamellipodia in developing neurons 
to regulate neuronal branching and growth (Higuero et al, 2010). We thus speculate that ARMS might have a role in the regulation of cytoskeleton and hence affect cell motility in both physiological and pathological processes, which definitely needs further experiments to prove the hypothesis.

\section{ACKNOWLEDGEMENTS}

We thank Ms Yu-Shan Tseng for statistical analysis. This study was supported by grants from National Science Council, Taiwan
(Project 98-2314-B-002-028-MY3, awarded to YHL), and National Taiwan University Hospital (Project NTUH98P26-2, awarded to $\mathrm{PHH}$ ); NTUH M0-23, awarded to YHL.

\section{Conflict of interest}

The authors declare no conflict of interest.

Supplementary Information accompanies the paper on British Journal of Cancer website (http://www.nature.com/bjc)

\section{REFERENCES}

Arevalo JC, Pereira DB, Yano H, Teng KK, Chao MV (2006) Identification of a switch in neurotrophin signaling by selective tyrosine phosphorylation. J Biol Chem 281: $1001-1007$

Arevalo JC, Yano H, Teng KK, Chao MV (2004) A unique pathway for sustained neurotrophin signaling through an ankyrin-rich membrane spanning protein. EMBO J 23: $2358-2368$

Balch CM, Gershenwald JE, Soong SJ, Thompson JF, Atkins MB, Byrd DR, Buzaid AC, Cochran AJ, Coit DG, Ding S, Eggermont AM, Flaherty KT, Gimotty PA, Kirkwood JM, McMasters KM, Mihm Jr MC, Morton DL, Ross MI, Sober AJ, Sondak VK (2009) Final version of 2009 AJCC melanoma staging and classification. J Clin Oncol 27: 6199-6206

Balch CM, Soong SJ, Gershenwald JE, Thompson JF, Reintgen DS, Cascinelli N, Urist M, McMasters KM, Ross MI, Kirkwood JM, Atkins MB, Thompson JA, Coit DG, Byrd D, Desmond R, Zhang Y, Liu PY, Lyman GH, Morabito A (2001) Prognostic factors analysis of 17600 melanoma patients: validation of the American Joint Committee on Cancer Melanoma Staging System. J Clin Oncol 19: $3622-3634$

Brocker EB, Magiera H, Herlyn M (1991) Nerve growth and expression of receptors for nerve growth factor in tumors of melanocyte origin. J Invest Dermatol 96: 662-665

Chang MS, Arevalo JC, Chao MV (2004) Ternary complex with Trk, p75, and an ankyrin-rich membrane spanning protein. J Neurosci Res 78: $186-192$

Dhillon AP, Rode J (1982) Patterns of staining for neuron specific enolase in benign and malignant melanocytic lesions of the skin. Diagn Histopathol 5: $169-174$

Easty DJ, Guthrie BA, Maung K, Farr CJ, Lindberg RA, Toso RJ, Herlyn M, Bennett DC (1995) Protein B61 as a new growth factor: expression of B61 and up-regulation of its receptor epithelial cell kinase during melanoma progression. Cancer Res 55: 2528-2532

Higuero AM, Sanchez-Ruiloba L, Doglio LE, Portillo F, Abad-Rodríguez J, Dotti CG, Iglesias T (2010) Kidins220/ARMS modulates the activity of microtubule-regulating proteins and controls neuronal polarity and development. J Biol Chem 285: $1343-1357$
Iglesias T, Cabrera-Poch N, Mitchell MP, Naven TJP, Rozengurt E, Schiavo G (2000) Identification and cloning of Kidins220, a novel neuronal substrate of protein kinase D. J Biol Chem 275: 40048-40056

Innominato PF, Libbrecht L, van den Oord JJ (2001) Expression of neurotrophins and their receptors in pigment cell lesions of the skin. J Pathol 194: 95 - 100

Kong H, Boulter J, Weber JL, Lai C, Chao MV (2001) An evolutionarily conserved transmembrane protein that is a novel downstream target of neurotrophin and ephrin receptors. J Neurosci 21: 176-185

Lammerding-Koppel M, Noda S, Blum A, Schaumburg-Lever G, Rassner G, Drews U (1997) Immunohistochemical localization of muscarinic acetylcholine receptors in primary and metastatic malignant melanomas. J Cutan Pathol 24: $137-144$

Liao YH, Hsu SM, Huang PH (2007) ARMS depletion facilitates UV irradiationinduced apoptotic cell death in melanoma. Cancer Res 67: 11547-11556

Miller AJ, Mihm Jr MC (2006) Melanoma. N Engl J Med 355: 51-65

Shonukan T, Bagayogo I, McCrea PD, Chao M, Hempstead B (2003) Neurotrophin-induced melanoma cell migration is mediated through the actin-bundling protein fascin. Oncogene 22: 3616-3623

Smalley KSM (2003) A pivotal role for ERK in the oncogenic behaviour of malignant melanoma. Int J Cancer 104: 527-532

Sober AJ, Fitzpatrick TB, Mihm MC, Wise TG, Pearson BJ, Clark WH, Kopf AW (1979) Early recognition of cutaneous melanoma. J Am Med Assoc 242: 2795 - 2799

Soltani MH, Pihardo R, Song Z (2005) Microtubule-associated protein 2, a marker of neuronal differentiation, induces mitotic defects, inhibits growth of melanoma cells, and predicts metastatic potential of cutaneous melanoma. Am J Pathol 166: 1841 - 1850

Walch ET, Albino AP, Marchetti D (1999) Correlation of overexpression of the low-affinity p75 neurotrophin receptor with augmented invasion and heparanase production in human malignant melanoma cells. Int J Cancer 82: $112-120$

Zhuang L, Lee CS, Scolyer RA, McCarthy SW, Palmer AA, Zhang XD, Thompson JF, Bron LP, Hersey P (2005) Activation of the extracellular signal regulated kinase (ERK) pathway in human melanoma. J Clin Pathol 58: $1163-1169$ 